\title{
MDP regulates BMSC differentiation and bone formation through miR-384-5p/Lepr
}

\section{Yun-Zhi Feng ( $\square$ fengyunzhi001@csu.edu.cn )}

Second Xiangya Hospital https://orcid.org/0000-0002-2498-6455

Shao-Hui Zhang

Second Xiangya Hospital

Yue Guo

Second Xiangya Hospital

Yun Chen

Second Xiangya Hospital

Qiong Liu

Second Xiangya Hospital

Li Tan

Second Xiangya Hospital

Zheng-Rong Gao

Second Xiangya Hospital

Jie Zhao

Second Xiangya Hospital

Ya-Qiong Zhao

Second Xiangya Hospital

Yao Feng

Second Xiangya Hospital

Jing $\mathrm{Hu}$

Second Xiangya Hospital

Qing Ye

Second Xiangya Hospital

Zhe-Yue Ou-Yang

Second Xiangya Hospital

Ying-Hui Zhou

Second Xiangya Hospital

\section{Research Article}

Keywords: MDP, BMSC differentiation, bone formation, miR-384-5p, Lepr 
Posted Date: March 1st, 2022

DOI: https://doi.org/10.21203/rs.3.rs-1376201/v1

License: (c) (i) This work is licensed under a Creative Commons Attribution 4.0 International License. Read Full License 


\section{Original Research}

MDP regulates BMSC differentiation and bone formation through miR-3845p/Lepr

Shao-Hui Zhang ${ }^{1 \#}$, Yue Guo ${ }^{1 \#}$, Yun Chen ${ }^{1}$, Qiong Liu ${ }^{1}$, Li Tan ${ }^{1}$, Zheng-Rong Gao ${ }^{1}$, Jie Zhao ${ }^{1}$, Ya-Qiong Zhao ${ }^{1}$, Yao Feng ${ }^{1}$, Jing $\mathrm{Hu}^{1}$, Qin $\mathrm{Ye}^{1}$, Zhe-Yue Ou-Yang ${ }^{1}$, Ying-Hui Zhou $^{2}$, Yun-Zhi Feng ${ }^{1 *}$.

${ }^{1}$ Department of Stomatology, The Second Xiangya Hospital, Central South University, Changsha, Hunan 410011, China

${ }^{2}$ Department of Metabolism \& Endocrinology, National Clinical Research Center for Metabolic Disease, The Second Xiangya Hospital, Central South University, Changsha, Hunan 410011, China

\#These authors contributed equally to this work.

Corresponding author: Yun-Zhi Feng, Department of Stomatology, The Second Xiangya Hospital, Central South University, Changsha, Hunan 410011, China, telephone: +8613574854398 , e-mail: fengyunzhi001@csu.edu.cn. 


\begin{abstract}
The main components of bacterial flora are closely related to bone metabolism. Peptidoglycan-based muramyl dipeptide (MDP) is present in bacterial cell walls. We found an association of energy metabolism with MDP in mice fed different diets. Leptin receptor (Lepr) expression in alveolar bone and the femur was increased drastically in the high-fat-diet group with MDP (H-MDP) compared with that in the high-fat-diet control $(\mathrm{H}-\mathrm{NaCl})$ group and low-fat-diet (LFD) group. The bone mass of mice was decreased after MDP intervention in the LFD group whereas, in the high-fat-diet (HFD) group, it was increased upon MDP intervention. Micro-RNA (miR)-384-5p targeted and inhibited Lepr expression in vitro. We extracted the supernatants of osteocytes cultured under different conditions with MDP intervention to act on bone marrow stromal cells (BMSCs) and found that miR-384-5p could regulate epr expression and osteogenic differentiation in BMSCs. Our findings indicate a possible mechanism of periodontitis-associated alveolar bone changes caused by the main components of microflora.
\end{abstract}

Keywords: MDP, BMSC differentiation, bone formation, miR-384-5p, Lepr

\author{
Abbreviations \\ MDP metabolism. Peptidoglycan-based muramyl dipeptide \\ Lepr Leptin receptor \\ BMSCs bone marrow stromal cells \\ cAMP cyclic adenosine monophosphate \\ ATP adenosine triphosphate \\ BAT brown adipose tissue \\ scWAT. subcutaneous white adipose tissue \\ vWAT visceral white adipose tissue \\ PTHrP PTH-related peptide \\ Irs-1 insulin receptor substrate-1 \\ HG high glucose \\ LG low glucose \\ ALP Alkaline phosphatase \\ $\mathrm{H} \& \mathrm{E} \quad$ hematoxylin and eosin \\ MLO-Y4 Murine osteocyte-like cells \\ HEK293T Human embryonic kidney 293 T \\ ANOVA Analysis of variance
}




\section{Introduction}

Periodontal disease is a chronic inflammatory disease characterized by the destruction of connective tissue and alveolar bone which, eventually, results in loss of gingival tissue, the periodontal ligament, cementum, and alveolar bone [1]. An epidemiological study involving 291,170 participants estimated that periodontitis was the sixth-most prevalent disease, affecting 743 million people, worldwide [2]. In the USA, periodontitis affects $47 \%$ of adults [3]. Periodontitis is the main cause of tooth loss in adults, which can affect their masticatory dysfunction, nutrition, and self-esteem [4]. Evidence does not support the notion that periodontitis has a simple linear relationship between bacterial accumulation and the initiation and severity of periodontal disease [5]. Oral bacteria induce bone loss by inducing gingival injury and immune stimulation [6]. Little is known about the specific bacterial components that may affect bone metabolism [7]. Therefore, additional research is needed to understand the role of microflora-associated factors in periodontitis.

Importantly, bacteria can change the host's metabolism. Muramyl dipeptide (MDP) is a peptidoglycan and is the main component of bacterial cell walls [8]. MDP can regulate expression of receptor activator of nuclear factor kappa-B ligand in osteoblasts through nucleotide-binding oligomerization domain-containing protein-2 (Nod-2), so it may play a key part in bone resorption in inflammatory diseases such as periodontitis [9]. In addition to causing oral diseases such as periodontitis, microflora can regulate disorders of the systemic metabolism. Studies have shown that microflora are involved in regulation of energy metabolism in the host [10]. Indigestible carbohydrates are fermented into short-chain fatty acids that can be utilized by the host. These short-chain fatty acids can affect the feeding behavior and energy metabolism of the host directly [11]. Butyric acid promotes intestinal gluconeogenesis in a free fatty acid receptor 2independent way, which results in higher adenosine triphosphate (ATP) levels and, in turn, higher levels of cyclic adenosine monophosphate (cAMP) [12]. Conversely, microflora are thought to inhibit expression of lipoprotein lipase by affecting the level of angiogenin-like protein- 4 in the circulation, thereby reducing the absorption of fatty acids by human tissues [13]. Moreover, the components of bacterial cell walls may play an important part in metabolism. Host detection of the components of bacterial cell walls is sufficient to promote the immune response, metabolic inflammation, lipolysis, and insulin resistance [14]. MDP injection has been shown to reduce adipose inflammation and glucose tolerance in obese mice, and MDP could reduce hepatic insulin resistance in obese mice and mice suffering from endotoxemia. Activating MDP of Nod1 can aggravate glucose tolerance [15].

Systemic energy metabolism is closely related to bone metabolism and maintenance of bone health [16]. Clinical studies have shown that the high-energy metabolism of hyperthyroidism can lead to secondary osteoporosis and reduction in bone mineral density, which is based on accelerated bone turnover and bone resorption [17]. However, observational studies have provided evidence that people with type-2 diabetes mellitus tend to have higher bone mineral density than people not suffering from diabetes mellitus [18]. The energy metabolism of bone is closely related to bone-marrow stromal cells (BMSCs). The latter are pluripotent and can differentiate into various cell types needed for tissue regeneration, including osteoblasts and adipocytes [19]. As cells related to energy metabolism, adipocytes are rich in mitochondria. They can regulate energy metabolism through the brown adipose tissue (BAT)-specific gene uncoupling protein-1 [20]. 
The main nutrient of BMSCs is glucose, which can be transported through glucose transporter-1. Glucose intake is beneficial to osteoblast differentiation and bone formation, thereby revealing the key regulatory role of metabolic requirements [21]. Studies have shown that osteocytes can regulate BMSC differentiation [22, 23]. By extracting the supernatants of osteocytes, we found that the exosomes from osteocytes carrying microRNA (miR)-124-3p could regulate galectin-3 expression in osteoblasts [24].

The leptin receptor (Lepr) belongs to a family of cytokine receptors [25]. In response to diet and obesity, the leptin receptor acts as a systematic "sensor" for energy homeostasis in BMSCs, which can promote fat formation and inhibit osteogenesis [26]. Liu et al. showed that subcutaneous injection of exogenous PTH-related peptide (PTHrP) can promote fracture repair in $\mathrm{Lepr}^{-1-}$ mice largely because of upregulation of expression of the genes and proteins in osteoblasts [27].

miRs are small regulatory non-coding RNAs of length $\sim 22$ bp. miRs regulate expression of post-transcriptional genes by identifying specific messenger (m)RNA sequences [28]. Abnormal expression of miRs during bone remodeling leads to pathologic bone resorption and bone diseases [29]. Previously, we showed that type-I collagen plays an important part in osteogenesis regulation through insulin receptor substrate-1 (Irs-1)/miR-342 and increases osteogenesis. This phenomenon is due to the differentiation of immature osteoblasts into mature osteoblasts and increased synthesis of collagen fibers [30].

In this study, we established a mouse model to study the effect of MDP on energy metabolism under different dietary conditions. We aimed to examine the mechanism of Lepr changes after MDP intervention. Hence, we measured lepr expression in BMSCs under high glucose (HG) and low glucose (LG) conditions, and its effect on osteogenic differentiation. 


\section{Materials and methods}

\section{Ethical approval of the study protocol}

The study protocol was approved by the Ethics Committee, the Second Xiang-Ya Hospital of Central South University (Changsha, China), conformed to the ARRIVE guidelines, and followed the Guiding Principles in the Care and Use of Animals endorsed by the China Physiological Society.

\section{Animals}

Twelve male C57BL/6J mice ( 4 weeks; $20-25$ g; Hunan SJA Laboratory Animals, Hunan, China) were housed in the Animal Experiment Center of Central South University under specific pathogen-free conditions; the animals had ad libitum access to food and water.

After 1 week of acclimatization, mice were randomly assigned into four groups $(n=3$ each): (a) low-fat diet control (L-NaCl), (b) low-fat diet with MDP (L-MDP), (c) highfat diet control (H-NaCl), and (d) high-fat diet with MDP (H-MDP).

For the high-fat-diet groups, separate cohorts of male mice were fed randomly for 8 weeks $(60 \% \mathrm{kcal}$ from fat, $20 \% \mathrm{kcal}$ from protein, $20 \% \mathrm{kcal}$ from carbohydrate; catalog number, TP23300; Trophic Animal Feed High-Tech, Nantong, China). For low-fat-diet groups, separate cohorts of male mice were fed randomly for 8 weeks $(45 \% \mathrm{kcal}$ from fat, $20 \% \mathrm{kcal}$ from protein, 35\% kcal from carbohydrate; TP23302; Trophic Animal Feed High-Tech).

The bodyweight of mice was monitored every day. The glucose level was determined by tail-vein blood sampling using a handheld glucometer (Sinocare, Changsha, China). In the last 10 days, MDP was injected (i.p.) every day. After the last injection of MDP, all mice were killed by anesthesia overdose. Then, blood, adipose tissue, and bone tissue were collected for further testing.

\section{Micro-computed tomography (micro-CT)}

The bone tissue of mice was fixed overnight in $10 \%$ paraformaldehyde solution at $4{ }^{\circ} \mathrm{C}$ and stored in $75 \%$ ethanol until use. Then, maxillary tissues were scanned by micro-CT (Siemens Medical Solutions, Knoxville, TN, USA). The sagittal plane of the mandible and femur of different groups of mice were taken to observe bone mass. Trabecular morphometry was characterized by measuring the fraction of bone volume/total volume (BV/TV), bone surface/bone volume (BS/BV), trabecular thickness (Tb.Th), trabecular number (Tb.N) and trabecular separation (Tb.Sp).

\section{Histology, staining, and immunohistochemistry}

Adipose tissue and bone tissue were fixed with 4\% paraformaldehyde solution for 2 days and decalcified in $10 \%$ EDTA (pH 7.4) for 21 days at $4^{\circ} \mathrm{C}$. Decalcified adipose tissue and bone tissue were embedded in paraffin, sectioned longitudinally at $5-\mu \mathrm{m}$ thickness, and mounted on glass slides. The paraffin sections of tissue were stained with hematoxylin and eosin (H\&E). Alkaline phosphatase (ALP) staining was undertaken with an ALP assay kit (Sigma-Aldrich, Saint Louis, MO, USA). Oil Red O staining was undertaken with an Oil Red O staining kit (Solarbio Life Sciences, Beijing, China) according to manufacturer instructions. Immunohistochemical staining for LepR was undertaken using goat anti-mouse Lepr antibody (R\&D Systems, Minneapolis, MN, 
USA). Antibody complexes were detected by diaminobenzidine (MaxVision ${ }^{\mathrm{TM}}$ HRPPolymer Anti-goat IHC Kit; Fuzhou Maixin Biotech, Fujian, China) and counterstained with hematoxylin before observation under a microscope (Zeiss, Oberkochen, Germany).

\section{RNA-seq analysis}

Following RNA isolation, the RNA was quantified (Qubit RNA Assay Kit; Life Technologies, Inc.), and the purity was assessed (RNA6000 Nano Kit and BioAnalyzer 2100; Agilent). Next, $1000 \mathrm{ng}$ RNA was used as the input for the VAHTSTM mRNA-seq v2 Library Prep Kit for Illumina ${ }^{\circledR}$ (Vazyme, Inc., Piscataway, NJ, USA), and sequencing libraries were created according to the manufacturer's protocol. Briefly, poly(A) RNA was purified in two rounds of hybridization to Dynal Oligo (dT) beads. Poly(A)+ RNA was fragmented and then used for first- and secondstrand cDNA synthesis with random hexamer primers. The cDNA fragments were treated with the End-It DNA EndRepair Kit to repair the ends, that were then modified with Klenow to add an A base at the $3^{\prime}$ end of the DNA fragments, and finally ligated to the adapters. The ligated cDNA products were subjected to PCR amplification. The library quality was determined using a Bioanalyzer 2100 (Agilent). The RNA-seq libraries were sequenced using the Illumina HiSeq TM 2000 platform. Based on the differentially expressed genes, GO functional significance enrichment analysis and KEGG pathway significant enrichment analysis were carried out.

\section{Enzyme-linked immunosorbent assay (ELISA)}

Blood samples from mice were centrifuged at $5000 \times g$ for $15 \mathrm{~min}$ and $4{ }^{\circ} \mathrm{C}$. The supernatant was stored at $-80^{\circ} \mathrm{C}$ for further separation of Lepr. According to the manufacturer instructions, an ELISA kit (Cusabio, Wuhan, China) was used to measure the protein concentration of Lepr in blood samples from mice.

\section{Western blotting}

Western blotting was performed as previously described (Chen, Y. et al., 2020). Briefly, cells were harvested, washed with phosphate-buffered saline, and lysed in RIPA buffer (which contained inhibitors of proteases and phosphatases). Then, the same amount of protein was separated by sodium dodecyl sulfate-polyacrylamide gel electrophoresis using $10 \%(w / v)$ gels and transferred (by electrophoretic means) to polyvinylidene fluoride (PVDF) membranes (0.22- $\mu$ m thickness; Millipore, Billerica, MA, USA). Next, PVDF membranes were incubated at $4{ }^{\circ} \mathrm{C}$ overnight with antibodies against Lepr (1;2000 dilution; AF497; R\&D Systems) and $\beta$-actin (1:5000; Proteintech, Rosemont, IL, USA). Immunoblotting with peroxidase-labeled secondary antibody (1:5000; Proteintech, Rosemont, IL, USA) was performed, and an ECL detection kit was used for visualization of chemiluminescence (Imager 600; Amersham, Buckinghamshire, UK). The density of protein bands was measured by ImageJ (US National Institutes of Health, Baltimore, MD, USA).

\section{Cell culture and transfection}

BMSCs were harvested by flushing the long bones of mice. Murine osteocyte-like cells (MLO-Y4) were purchased from the Cell Bank of the Chinese Academy of Sciences (Shanghai, China). Before intervention, cells were cultured in $\alpha$-minimum essential medium supplemented with 10\% fetal bovine serum (Gibco, Grand Island, NY, USA), penicillin $(100 \mathrm{U} / \mathrm{mL})$ and streptomycin $(100 \mu \mathrm{g} / \mathrm{mL})$. For intervention with a high glucose concentration, Dulbecco's modified Eagle's medium-high glucose (DMEM- 
HG; $4.5 \mathrm{~g} / \mathrm{L}$ ) was used. For intervention using a low concentration of glucose, DMEMLG $(1.5 \mathrm{~g} / \mathrm{L})$ was used. All cells were stored in a humidified incubator in an atmosphere of $5 \% \mathrm{CO}_{2}$ at $37^{\circ} \mathrm{C}$. Liposome 2000 (27623927; Thermo Fisher Scientific, Waltham, MA, USA) was used for cell transfection. Briefly, BMSCs were seeded in six-well plates, and then transfected with a miR-384-5p mimic and its negative control (miR384-5p mimic NC) and a miR-384-5p inhibitor and its negative control (miR-384-5p inhibitor NC) (RiboBio, Guangzhou, China).

\section{Osteogenic differentiation and adipogenic differentiation}

For osteogenic differentiation, $2 \times 10^{6}$ cells/well were seeded in six-well plates in differentiation medium comprising osteo-differentiation medium (dexamethasone [10 $\mathrm{nM}], \beta$-glycerophosphate $[10 \mu \mathrm{mol} / \mathrm{L}]$, and L-ascorbic acid $[50 \mu \mathrm{g} / \mathrm{mL}])$ with dexamethasone $(100 \mathrm{nM})$. Treatment was administered for 3 days, and the medium was changed on day 2. At the end of the experiment, cells were washed in PBS and stained with the ALP Assay Kit.

For adipogenic differentiation, $1 \times 10^{4}$ cells/well were seeded in six-well plates and cultured in a complete growth medium. At $100 \%$ confluence, the medium was changed to adipo-differentiation medium (3-isobutyl-1-methylxanthine [0.5 $\mathrm{mM}]$, indomethacin $[100 \mu \mathrm{M}]$, insulin $[10 \mu \mathrm{g} / \mathrm{mL}]$, and dexamethasone $[1 \mu \mathrm{M}])$. Adipocyte differentiation was identified by the presence of fat globules visualized by the Oil Red O Staining Kit.

\section{Real-time reverse transcription-quantitative polymerase chain reaction (RT- qPCR)}

Real-time RT-qPCR was carried out according to protocols published previously [24]. RNA extraction from cells was performed using TRIzol ${ }^{\circledR}$ Reagent according to manufacturer (Thermo Fisher Scientific) instructions. RNA was reverse-transcribed using a PrimeScript RT Reagent Kit and gDNA Eraser (TaKaRa Biotechnology, Shiga, Japan). Amplification and detection were undertaken with TB Green Premix Ex Taq II (Tli RNaseH Plus) reagent (TaKaRa Biotechnology) and the LightCycler ${ }^{\text {TM }}$ Real-time PCR system (Roche, Basel, Switzerland). The primer sequences (forward and reverse, respectively) were 5'-CAACGAGCGGTTCCGATG-3' and 5'GCCACAGGATTCCATACCCA-3' for $\beta$-actin, and 5'-GAGCAGGCGTGCCATC-3', and 5'-GTACCCGTCAGTTTCACATGATATATTG-3' for Lepr. For miRs, the RNA reverse-transcription reaction was carried out with a PrimeScript miRNA quantitative PCR Starter Kit (TaKaRa Biotechnology) and relative miR expression was normalized to that of U6 (TaKaRa Biotechnology).

\section{Dual luciferase reporter assay}

Human embryonic kidney 293T (HEK293T) cells were inoculated on a 24-well plate and grown to $70-80 \%$ confluence before transfection. To examine luciferase reporter activity, Lepr-3' untranslated region (UTR) (WT) and Mut-Lepr-3' UTR (MUT) were inserted into psiCHECK-2 (luciferase expression vector). Then, the two vectors were co-transfected into HEK293T cells (NC) or with miR-384-5p mimics, miR-384-5p inhibitor, or NC using Endofectin ${ }^{\mathrm{TM}}$-MAX Reagent (EF004; GeneCopoeia, Rockville, ML, USA). After $48 \mathrm{~h}$ of incubation, HEK293T cells were analyzed for luciferase activity with a dual-luciferase reporter assay system (E1910; Promega, Madison, WI, USA). The relative luciferase signal was represented by the ratio of firefly luciferase activity to that of the sea kidney. 


\section{Statistical analyses}

Data are represented as the mean \pm SD. Experiments were conducted in triplicate. The significance of differences between groups was analyzed by one-way analysis of variance or the Student's $t$-test. $\mathrm{P}<0.05$ (two-tailed) was considered significant. 


\section{Results}

\section{MDP regulates diet-induced glucose homeostasis and adipogenesis}

To examine in detail the effects of MDP on energy metabolism in mice, we used two experimental models of different diets (Figure 1A, B). Model 1 involved feeding a highfat diet (HFD) and injection with MDP daily (10 days before sacrifice), and Model 2 involved feeding a low-fat diet (LFD) and injection with MDP daily (10 days before sacrifice). In both regimens, the final MDP injection was given $24 \mathrm{~h}$ before measurement of metabolic and inflammatory phenotypes. In the HFD model, the bodyweight of mice increased, but the blood glucose level after MDP injection was unaltered (Figure 1C, E). However, in the LFD model, MDP injections could reduce the bodyweight and blood glucose level of mice after 9 weeks on an LFD (Figure 1D, F).

Briefly, H\&E staining of interscapular brown adipose tissue (iBAT) revealed that more lipid droplets, smaller parenchyma, and infiltration of more inflammatory cells were observed in the LFD group after MDP injection. However, in the HFD model, MDP injection reduced the number of inflammatory cells, and we noted enlargement of lipid droplets. Then, in the LFD model, the subcutaneous white adipose tissue (scWAT) inflammatory cells were decreased and the lipid droplets became lager after MDP injection. In the HFD model, the number of adipocytes was increased, the number of lipid droplets was increased, and the inflammatory reaction was more robust. Interestingly, in the visceral white adipose tissue (vWAT) group, the mean number of adipocytes in the HFD group was greater than that in the LFD group, and there was no significant difference in the same dietary group (Figure $1 \mathrm{G}$ ).

\section{Lepr participates in the changes of energy metabolism induced by MDP}

We wished to further explore the mechanism of MDP-induced changes in glucose homeostasis and adipogenesis in mice. We identified differentially expressed genes related to energy metabolism using high-throughput PCR arrays.

We discovered that multiple genes related to energy metabolism were affected by MDP intervention. We found significant changes in expression of 12 genes related to metabolism. Among these 12 genes, expression of eight genes was upregulated such as Acs16,Tbl1xr1, and expression of four genes was downregulated such as Acacb, among which lepr expression was upregulated significantly ( $>9.0$-fold change) (Figure $2 \mathrm{~A}$ ). Analyses of signaling-pathway enrichment using the Kyoto Encyclopedia of Genes and Genomes (KEGG) database revealed enrichment in "Metabolic pathways," "MAPK signaling pathway," "TNF signaling pathway," and "Transcriptional misregulation." Among them, the change in metabolic pathways was the most obvious (Figure 2B). Analyses of functional enrichment using the Gene Ontology (GO) database showed that these differentially expressed genes were mainly enriched in "cellular process," "singleorganism process," and "metabolic process"; "cell," "cell part and organelle"; and "binding, catalytic activity and transporter activity" (Figure 2C).

We further verified the changes of Lepr expression in our animal model. The ELISA results for Lepr expression in serum are shown in Figure 2D. In the LFD group, Lepr expression upon MDP injection was increased compared with that in the control group. In the HFD group, there was no significant difference between the two groups for Lepr expression. 
Protein expression of Lepr in the adipose tissue, alveolar bone, and femur of mice was measured using immunohistochemistry (Figure 2E, F). Among the three types of adipose tissue, Lepr expression in LFD mice injected with MDP was increased more than that in the NC group; however, interestingly, Lepr expression in HFD mice was decreased more than that in the $\mathrm{NC}$ group. In the alveolar bone and femur, compared with that in the NC group, LFD mice and HFD mice injected with MDP had increased Lepr expression in osteoblasts, BMSCs, and osteocytes. Expression of Lepr protein was augmented in HFD mice upon MDP injection compared with that in the other three groups.

\section{Effect of MDP on the mass and quality of bone in male mice consuming an HFD or LFD}

Three-dimensional images of the alveolar bone and femur of mice in the L-NaCL, L$\mathrm{MDP}, \mathrm{H}-\mathrm{NaCl}$, and $\mathrm{H}-\mathrm{MDP}$ groups were constructed using micro-CT (Figure 3A, B). In the mandible and femur, L-MDP mice had decreased bone mass compared with L$\mathrm{NaCL}$ mice. BV/TV and Tb.Th were decreased in the L-MDP compared with L-NaCl mice. In the $\mathrm{H}-\mathrm{MDP}$ group compared with the $\mathrm{H}-\mathrm{NaCl}$ group, $\mathrm{BV} / \mathrm{TV}$ and $\mathrm{Tb} . \mathrm{N}$ were increased and Tb.Sp was decreased in the mandible and femur. However, in the mandible and femur, we found Tb.Th had no statistical significance, in the H-MDP group compared with the $\mathrm{H}-\mathrm{NaCl}$ group. In the mandible and femur, we noted Tb.N and Tb.sp had no statistical significance in the L-MDP group compared with the L$\mathrm{NaCl}$ group.

In the L-MDP group, H\&E staining of the femur showed that trabeculae became thinner and fewer in number than in the L-NaCl group, and scattered adipocytes could be seen. In the H-MDP group, trabeculae became thicker and more numerous than in the LFD group, and fewer adipocytes could be observed.

miR-384-5p contained the targets of Lepr and modulated Lepr expression in vitro The binding target sequences of mmu-miR-384-5p and Lepr-3'UTR were predicted using a miRNA database (Figure 4A). As described above, Lepr-WT and Lepr-MUT vectors were constructed. Figure $4 \mathrm{~B}$ shows the sequences of the two vectors (red rectangle highlights the binding site in WT and core mutation site in MUT). After transfection of HEK293T cells with these constructs, luciferase activity was inhibited by the miR-384-5p mimic in the Lepr-WT group but showed no difference in the LeprMUT group (Figure 4D).

Next, expression of miR-384-5p and Lepr was measured at day 0 and day 3 of osteogenic differentiation of BMSCs. During osteogenic differentiation of BMSCs, Lepr expression showed an increasing trend, but miR-203 expression was decreased (Figure 4E).

To further validate that miR-384-5p modulated Lepr expression, we undertook transfection with the miR-384-5p mimic and miR-384-5p inhibitor and their respective NCs in BMSCs. miR-384-5p expression increased after transfection of the miR-384-5p mimic and decreased by $\sim 80 \%$ after induction of miR-384-5p inhibition in BMSCs. Conversely, expression of Lepr mRNA decreased after transfection of the miR-384-5p mimic but increased after miR-384-5p inhibition in BMSCs (Figure 4F). 


\section{miR-384-5p affected osteogenic differentiation of BMSCs through regulation of Lepr expression}

To further explore expression of miR-384-5p and Lepr in bone metabolism induced by MDP, we simulated the HFD environment with a HG medium and the LFD environment with a LG medium. We simulated osteocytes and BMSCs in vitro. As described in our previous research [24], we first cultured osteocytes in the two abovementioned culture environments, and the supernatant was extracted after $36 \mathrm{~h}$ of intervention with MDP. Next, the extracted supernatants were assigned into four groups: (a) LG medium control (L-NC), (b) LG medium with MDP (L-MDP), (c) HG medium control (H-NC), and (d) HG medium with MDP (H-MDP). Next, we used the supernatants of the four groups stated above to interfere with the osteogenic and adipogenic induction of BMSCs, and carried out related ALP staining or Oil Red O staining in BMSCs (Figure 5A). The ALP content in the L-MDP group was increased compared with that in L-NC group. Likewise, the ALP content in the H-MDP was enhanced compared with that in the H-NC group. ALP staining was strongest in the HMDP group. Interestingly, the result of Oil Red $\mathrm{O}$ staining after adipogenic induction of BMSCs was exactly the opposite to that observed with ALP staining.

We also measured expression of Lepr and miR-384-5p after interventions using extracted supernatants in BMSCs (Figure 5B, C). Expression of Lepr protein was consistent with that of the mRNA expression. Lepr expression increased with MDP induction when compared with that in their respective NC groups. In the H-MDP group, Lepr expression was the highest among the four groups mentioned above. Based on the RT-qPCR results, miR-384-5p expression showed the opposite results compared to Lepr expression. Our results implied that miR-384-5p affected osteogenic differentiation by regulation of Lepr expression. 


\section{Discussion}

We detected changes in the blood glucose level, body weight, and adipose tissue of mice on different diets, with or without MDP, and found an association of energy metabolism with MDP. We also observed drastically increased Lepr expression in the alveolar bone and femur in the H-MDP group compared with that in the $\mathrm{H}-\mathrm{NaCl}$ group and LFD group. However, expression of Lepr in adipose tissue was opposite to that in bone tissue. Moreover, the bone mass of mice was decreased after MDP intervention in the LFD group, whereas, in the HFD group, the bone mass of mice increased upon MDP intervention. Finally, we used MDP to interfere with osteocytes cultured under HG and LG conditions, and extracted supernatants to act on BMSCs. We found that miR-384$5 p$ could regulate Lepr expression and osteogenic differentiation in BMSCs. This result suggested an important role for miR-384-5p in regulating the energy metabolism and bone formation of mice after MDP intervention.

According to previously described protocols [31], the final MDP injection was administered $24 \mathrm{~h}$ before phenotyping of metabolic and inflammatory mediators. In the HFD model, there was no significant change in the blood glucose levels of mice. In the LFD model, MDP injection could reduce the blood glucose level. This result is consistent with observations from studies indicating that inflammation in an animal model is also induced by HFD [32]. In addition, excessive intake of calories usually leads to glucose intolerance and insulin resistance [33].

Mammals have two types of adipose tissue: WAT and BAT [34]. Some commonly studied WATs in rodent models are scWAT and vWAT [35]. WAT stores energy in the form of lipids. BAT uncouples oxidative phosphorylation with ATP production through brown adipocyte-specific uncoupling protein-1, and consumes energy directly in the form of calories [36]. BAT can also improve insulin sensitivity and regulate glucose homeostasis [37].

H\&E staining of iBAT showed that the energy supply of adipose tissue increased after MDP intervention in the LFD group, but decreased in the HFD group. However, in the scWAT group, fat droplets became larger and energy storage of adipose tissue increased after MDP intervention in the LFD group, whereas, in the HFD group, "browning" of white fat (which can resist obesity-related harm) was observed. In the WAT group, there was no significant difference between the LFD group and HFD group after MDP intervention, but the LFD group had smaller fat droplets and infiltration of more inflammatory cells, which revealed a healthier state than the HFD group. Owing to the heterogeneity and plasticity of adipocytes, the morphology and function of white adipocytes and brown adipocytes changes if the energy demand changes [38, 39]. Brown adipocytes develop and appear in WAT (browning) [40]. In the LFD group after MDP intervention, the energy supply of BAT decomposition would be greater than that of WAT which, in turn, would promote the energy consumption and weight loss of mice. In contrast, HFD is associated with a state of inflammation, which would cause disorder of the metabolic function of mice. Hence, MDP injection seemed to change the energy metabolism of mice and be more beneficial in the LFD group. Therefore, we attempted to analyze the reason for this effect.

Studies have shown that osteocytes can stimulate the osteogenesis of BMSCs. If BMSCs are co-cultured with osteocytes, the key indicators of osteogenic differentiation - calcium deposition and ALP levels - are increased significantly[22]. 
Previously, we showed that MDP was beneficial to LFD mice, and we focused on its beneficial mode of action. Hence, we examined the protective effect of MDP intervention on the health of mice under normal conditions. Therefore, we used MDP to interfere with osteocytes and identified differentially expressed genes related to energy metabolism by high-throughput PCR arrays: Lepr expression was increased significantly after MDP intervention. We measured Lepr expression in serum and found that MDP intervention increased Lepr expression in the LFD group, but there was no significant change in the HFD group. Leptin acts as a mediator of inflammation and energy balance by activating Lepr [41]. Studies have shown changes in production of leptin and its receptor during acute infection and inflammation [42]. Gregor et al. showed that obese mice on an HFD had chronic low-grade inflammation of adipose tissue and the liver [43]. The binding of Lepr and leptin in various peripheral tissues is involved in lipid metabolism, pancreatic-cell function, hematopoiesis, and thermogenesis [44, 45]. Hence, there may have been a dynamic imbalance of Lepr expression in the HFD group, which resulted in no significant change of Lepr expression in the blood circulation.

In addition to determining Lepr expression in the serum of mice treated with MDP under different dietary conditions, we also found that Lepr expression in osteocytes increased. Simultaneously, because of regulation of osteoblasts and BMSCs by osteocytes, we also focused on Lepr expression in these two types of cells. Lepr expression was also increased. Expression of Lepr protein in the LFD group treated with MDP showed strong immunohistochemical staining, which was consistent with the results of our high-throughput PCR arrays (Figure 2F). These data suggested that we should pay attention to the beneficial mode of action of MDP, and that a combination of leptin and its receptor is involved in regulation of energy metabolism.

L-MDP mice had a reduction of bone mass compared with that in L-NaCL mice. However, in the HFD group, the bone mass of the MDP-intervention group was higher than that of the control group. This phenomenon may be related to the multi-lineage potential of BMSCs [46]. If the balance between adipocyte differentiation and osteoblast differentiation is disrupted, adipocyte differentiation is increased compared with osteoblast differentiation, which is related to decreased bone mass, increased bone brittleness, and increased susceptibility to fracture [47]. We found that the LFD group was in good health, and that the energy supply after iBAT decomposition increased after MDP intervention (Figure 1G), which led to a decrease in the energy supply of bone tissue, and resulted in the weakening of osteogenesis and a relative decrease in bone mass. In the H-MDP group, mice were unhealthy, and the lipid droplets in iBAT were larger after MDP treatment, suggesting that adipose tissue was reduced to provide energy for decomposition, so bone tissue was needed for additional energy supply.

Bone is an endocrine organ that can regulate metabolism and energy balance during osteogenesis [48, 49]. Hence, an increase in the energy supply of bone tissue resulted in osteogenesis enhancement, which also led to a relative increase in bone mass in the H-MDP group. In the LFD group, more adipocytes were seen in H\&E-stained femurs after MDP intervention (indicating that adipogenic differentiation of BMSCs was enhanced), whereas, in the HFD group, adipogenic differentiation was decreased after MDP intervention (indicating that adipogenic differentiation of BMSCs was reduced). These data are consistent with our abovementioned results. Morrison et al. demonstrated that Lepr is a sensor for whole-body energy homeostasis, because it 
regulates BMSCs, promotes lipogenesis, and inhibits osteogenesis in response to diet and obesity [26]. Therefore, MDP intervention may be through a change in Lepr expression, which then leads to changes in bone metabolism.

Bioinformatics analysis and Dual luciferase reporter assay revealed that miR-384-5p targets Lepr through expression of specific binding sites. miR-384-5p transfection showed that miRNA could inhibit Lepr expression, and that MDP intervention could promote Lepr expression by inhibiting mir-384-5p expression during the osteogenic induction of BMSCs. Consistent with the abovementioned animal-model experiments, Lepr expression in serum and bone tissue increased after MDP intervention. In adipose tissue, expression of Lepr protein was inhibited in the H-MDP group, likely due to the impact of a more inflammatory environment.

Osteocytes can regulate the multi-lineage potential of BMSCs [22]. To further explore how MDP regulates bone formation, we extracted the supernatants of osteocytes after MDP intervention, and interfered with the osteogenic differentiation and adipogenic differentiation of BMSCs. We simulated the HFD environment with an HG medium and the LFD environment with an LG medium in the MLO-Y4 (for osteocytes) cell line. After that, we used the above four groups of supernatants to interfere with the osteogenic and adipogenic induction processes of BMSCs. The ALP content in the LG group intervened with MDP was increased more than that in the NC group, and a similar effect was observed in the HG group. Lepr was expressed on the surface of BMSCs [50] and leptin stimulation of BMSCs in vitro was shown to promote mineralization and partly inhibit adipogenic differentiation by binding to Lepr [51] Therefore, MDP intervention could increase Lepr expression and further promote its ability to enhance the osteogenic differentiation of BMSCs, which also meant that the ability of adipogenic differentiation of BMSCs decreased. Moreover, expression of Lepr protein and mRNA in the four groups of BMSC cells revealed that the inhibitory effect of MDP on miR-384-5p promoted Lepr expression which, in turn, enhanced the osteogenicdifferentiation ability of BMSCs.

However, we did not directly evaluate how the supernatants of osteocytes acted on BMSCs after MDP intervention, which may be the result of extracellular-vesicle transduction of miRs on BMSCs [52]. Recent studies have shown that miR-185 $5^{-1-}$ mice can promote osteogenic differentiation in osteoporosis through the BMP/Smad pathway and inhibit bone loss, thereby revealing the effect of miR inhibition on bone protection [53]. Hence, conditional knockout of miR-384-5p expression may need to be improved further. 


\section{Conclusions}

The supernatants of osteocytes treated with MDP may regulate Lepr expression in BMSCs through miR-384-5p, and then affect the osteogenic differentiation of BMSCs. Our findings indicate a possible mechanism of periodontitis-associated alveolar bone changes caused by the main components of microflora.

Acknowledgments This study was supported by the National Natural Science Foundation of China (81773339 and 81800788), Science and Technology Department of Hunan Province, China (2017WK2041 and 2018SK52511), Scientific Research Project of Hunan Provincial Health Commission (202208043514), Fund for the Xiangya Clinical Medicine Database of Central South University (2014-ZDYZ-1-16), Education and Teaching Reform Research Project of Central South University(2020jy 165-3), Research Project on Postgraduate Education and Teaching Reform of Central South University(2021 NO.61), Open Sharing Fund for the Largescale Instruments and Equipment of Central South University and the Fundamental Research Funds for the Central Universities of Central South University.

Author contributions Yun-Zhi Feng designed and conducted the study. Shao-Hui Zhang and Yue Guo wrote the manuscript and conducted the animal experiments and cell-culture experiments. Shao-Hui Zhang conducted western blotting and RT-qPCR experiments. Yun Chen and Qiong Liu conducted micro-CT experiments. Li Tan, Zheng-Rong Gao, Jie Zhao and Ya-Qiong Zhao undertook bioinformatics analysis. Yao Feng, Jing Hu and Qin Ye conducted tissue experiments. Ying-Hui Zhou used computer software to record experiments. The first draft of the manuscript was written by ShaoHui Zhang and Yue Guo and all authors commented on previous versions of the manuscript. All authors read and approved the final manuscript

Funding This study was supported by the National Natural Science Foundation of China (81773339 and 81800788), Science and Technology Department of Hunan Province, China (2017WK2041 and 2018SK52511), Scientific Research Project of Hunan Provincial Health Commission (202208043514) , Fund for the Xiangya Clinical Medicine Database of Central South University (2014-ZDYZ-1-16), Education and Teaching Reform Research Project of Central South University(2020jy165-3), Research Project on Postgraduate Education and Teaching Reform of Central South University(2021 NO.61), Open Sharing Fund for the Large-scale Instruments and Equipment of Central South University and the Fundamental Research Funds for the Central Universities of Central South University.

\section{Declarations}

Conflict of interes The authors have no relevant financial or non-financial interests to disclose. 


\section{References}

1. Loomer PM: Microbiological diagnostic testing in the treatment of periodontal diseases. Periodontol 2000 2004, 34:49-56.http://dx.doi.org/10.1046/j.0906-6713.2002.003424.x

2. Richards D: Review finds that severe periodontitis affects $11 \%$ of the world population. Evid Based Dent 2014, 15(3):70-71.http://dx.doi.org/10.1038/sj.ebd.6401037

3. Kassebaum NJ, Bernabé E, Dahiya M, Bhandari B, Murray CJ, Marcenes W: Global burden of severe periodontitis in 1990-2010: a systematic review and meta-regression. J Dent Res 2014, 93(11):1045-1053.http://dx.doi.org/10.1177/0022034514552491

4. Tonetti MS, Jepsen S, Jin L, Otomo-Corgel J: Impact of the global burden of periodontal diseases on health, nutrition and wellbeing of mankind: A call for global action. J Clin Periodonto/ 2017, 44(5):456-462.http://dx.doi.org/10.1111/jcpe.12732

5. Kornman KS: Contemporary approaches for identifying individual risk for periodontitis. Periodontol 2000 2018, 78(1):12-29.http://dx.doi.org/10.1111/prd.12234

6. Tanaka T, Kishimoto T: Targeting interleukin-6: all the way to treat autoimmune and inflammatory diseases. Int J Biol Sci 2012, 8(9):12271236.http://dx.doi.org/10.7150/ijbs.4666

7. Wolf AJ, Reyes CN, Liang W, Becker C, Shimada K, Wheeler ML, Cho HC, Popescu NI, Coggeshall KM, Arditi $\mathrm{M}$ et al. Hexokinase Is an Innate Immune Receptor for the Detection of Bacterial Peptidoglycan. Cell 2016, 166(3):624636.http://dx.doi.org/10.1016/j.cell.2016.05.076

8. Williams L, Alshehri A, Robichaud B, Cudmore A, Gagnon J: The Role of the Bacterial Muramyl Dipeptide in the Regulation of GLP-1 and Glycemia. Int J Mol Sci 2020, 21(15).http://dx.doi.org/10.3390/ijms21155252

9. Yang S, Takahashi N, Yamashita T, Sato N, Takahashi M, Mogi M, Uematsu T, Kobayashi Y, Nakamichi Y, Takeda K et al: Muramyl dipeptide enhances osteoclast formation induced by lipopolysaccharide, IL-1 alpha, and TNF-alpha through nucleotide-binding oligomerization domain 2-mediated signaling in osteoblasts. J Immunol 2005, 175(3):1956-1964.http://dx.doi.org/10.4049/jimmunol.175.3.1956

10. Woting A, Blaut M: The Intestinal Microbiota in Metabolic Disease. Nutrients 2016, 8(4):202.http://dx.doi.org/10.3390/nu8040202

11. Shipman JA, Berleman JE, Salyers AA: Characterization of four outer membrane proteins involved in binding starch to the cell surface of Bacteroides thetaiotaomicron. J Bacteriol 2000, 182(19):5365-5372.http://dx.doi.org/10.1128/jb.182.19.5365-5372.2000

12. Wang A, Si H, Liu D, Jiang $\mathrm{H}$ : Butyrate activates the cAMP-protein kinase A-cAMP response element-binding protein signaling pathway in Caco-2 cells. J Nutr 2012, 142(1):1-6.http://dx.doi.org/10.3945/jn.111.148155

13. Lichtenstein L, Kersten S: Modulation of plasma TG lipolysis by Angiopoietin-like proteins and GPIHBP1. Biochim Biophys Acta 2010, 1801(4):415420.http://dx.doi.org/10.1016/j.bbalip.2009.12.015

14. Schertzer JD, Tamrakar AK, Magalhães JG, Pereira S, Bilan PJ, Fullerton MD, Liu Z, Steinberg GR, Giacca A, Philpott DJ et al: NOD1 activators link innate immunity to insulin resistance. Diabetes 2011, 60(9):2206-2215.http://dx.doi.org/10.2337/db11-0004

15. Rafalski VA, Mancini E, Brunet A: Energy metabolism and energy-sensing pathways in 
mammalian embryonic and adult stem cell fate. J Cell Sci 2012, 125(Pt 23):55975608.http://dx.doi.org/10.1242/jcs.114827

16. Mittapalli S, Sravanakumar Perumalla D, Nangia A: Mechanochemical synthesis of Nsalicylidene-aniline: thermosalient effect of polymorphic crystals. IUCrJ 2017, 4(Pt 3):243250.http://dx.doi.org/10.1107/s2052252517004043

17. Tsourdi E, Rijntjes E, Köhrle J, Hofbauer LC, Rauner M: Hyperthyroidism and Hypothyroidism in Male Mice and Their Effects on Bone Mass, Bone Turnover, and the Wnt Inhibitors Sclerostin and Dickkopf-1. Endocrinology 2015, 156(10):35173527.http://dx.doi.org/10.1210/en.2015-1073

18. Schwartz AV, Vittinghoff E, Bauer DC, Hillier TA, Strotmeyer ES, Ensrud KE, Donaldson MG, Cauley JA, Harris TB, Koster A et al: Association of BMD and FRAX score with risk of fracture in older adults with type 2 diabetes. Jama 2011, 305(21):21842192.http://dx.doi.org/10.1001/jama.2011.715

19. Wan Y: Bone marrow mesenchymal stem cells: fat on and blast off by FGF21. Int J Biochem Cel/ Bio/ 2013, 45(3):546-549.http://dx.doi.org/10.1016/j.biocel.2012.12.014

20. You Y, Yuan X, Liu X, Liang C, Meng M, Huang Y, Han X, Guo J, Guo Y, Ren C et al: Cyanidin-3-glucoside increases whole body energy metabolism by upregulating brown adipose tissue mitochondrial function. Mol Nutr Food Res 2017, 61(11).http://dx.doi.org/10.1002/mnfr.201700261

21. Wei J, Shimazu J, Makinistoglu MP, Maurizi A, Kajimura D, Zong H, Takarada T, Lezaki T, Pessin JE, Hinoi E et al: Glucose Uptake and Runx2 Synergize to Orchestrate Osteoblast Differentiation and Bone Formation. Cell 2015, 161(7):15761591.http://dx.doi.org/10.1016/j.cell.2015.05.029

22. Birmingham E, Niebur GL, McHugh PE, Shaw G, Barry FP, McNamara LM: Osteogenic differentiation of mesenchymal stem cells is regulated by osteocyte and osteoblast cells in a simplified bone niche. Eur Cell Mater 2012, 23:1327.http://dx.doi.org/10.22203/ecm.v023a02

23. Liao C, Zhang C, Jin L, Yang Y: IL-17 alters the mesenchymal stem cell niche towards osteogenesis in cooperation with osteocytes. J Cell Physiol 2020, 235(5):44664480.http://dx.doi.org/10.1002/jcp.29323

24. Li J, Guo Y, Chen YY, Liu Q, Chen Y, Tan L, Zhang SH, Gao ZR, Zhou YH, Zhang GY et al: miR-124-3p increases in high glucose induced osteocyte-derived exosomes and regulates galectin- 3 expression: A possible mechanism in bone remodeling alteration in $\begin{array}{llll}\text { diabetic } \quad \text { periodontitis. Faseb } \quad \text { 2020, 34(11):14234- } & \end{array}$ 14249.http://dx.doi.org/10.1096/fj.202000970RR

25. Friedman JM, Halaas JL: Leptin and the regulation of body weight in mammals. Nature 1998, 395(6704):763-770.http://dx.doi.org/10.1038/27376

26. Yue R, Zhou BO, Shimada IS, Zhao Z, Morrison SJ: Leptin Receptor Promotes Adipogenesis and Reduces Osteogenesis by Regulating Mesenchymal Stromal Cells in Adult Bone Marrow. Cel/ Stem Ce//2016, 18(6):782-796.http://dx.doi.org/10.1016/j.stem.2016.02.015

27. Liu A, Li Y, Wang Y, Liu L, Shi H, Qiu Y: Exogenous Parathyroid Hormone-Related Peptide Promotes Fracture Healing in Lepr(-/-) Mice. Calcif Tissue Int 2015, 97(6):581591.http://dx.doi.org/10.1007/s00223-015-0041-2

28. Tang L, Yin Y, Liu J, Li Z, Lu X: MiR-124 Attenuates Osteoclastogenic Differentiation of 
Bone Marrow Monocytes Via Targeting Rab27a. Cell Physiol Biochem 2017, 43(4):16631672.http://dx.doi.org/10.1159/000484027

29. Liu X, Chin JF, Qu X, Bi H, Liu Y, Yu Z, Zhai Z, Qin A, Zhang B, Dai M: The Beneficial Effect of Praeruptorin $C$ on Osteoporotic Bone in Ovariectomized Mice via Suppression of Osteoclast Formation and Bone Resorption. Front Pharmacol 2017, 8:627.http://dx.doi.org/10.3389/fphar.2017.00627

30. Guo Y, Tang CY, Man XF, Tang HN, Tang J, Wang F, Zhou CL, Tan SW, Feng YZ, Zhou HD: Insulin receptor substrate-1 time-dependently regulates bone formation by controlling collagen la2 expression via miR-342. Faseb j 2016, 30(12):42144226.http://dx.doi.org/10.1096/fj.201600445RR

31. Cavallari JF, Fullerton MD, Duggan BM, Foley KP, Denou E, Smith BK, Desjardins EM, Henriksbo BD, Kim KJ, Tuinema BR et al: Muramyl Dipeptide-Based Postbiotics Mitigate Obesity-Induced Insulin Resistance via IRF4. Cell Metab 2017, 25(5):10631074.e1063.http://dx.doi.org/10.1016/j.cmet.2017.03.021

32. Jang HH, Nam SY, Kim MJ, Kim JB, Choi JS, Kim HR, Lee YM: Agrimonia pilosa Ledeb. aqueous extract improves impaired glucose tolerance in high-fat diet-fed rats by decreasing the inflammatory response. BMC Complement Altern Med 2017, 17(1):442.http://dx.doi.org/10.1186/s12906-017-1949-z

33. Kadowaki T, Hara K, Yamauchi T, Terauchi Y, Tobe K, Nagai R: Molecular mechanism of insulin resistance and obesity. Exp Biol Med (Maywood) 2003, 228(10):11111117.http://dx.doi.org/10.1177/153537020322801003

34. Gil A, Olza J, Gil-Campos M, Gomez-Llorente C, Aguilera CM: Is adipose tissue metabolically different at different sites? Int J Pediatr Obes 2011, 6 Suppl 1:1320.http://dx.doi.org/10.3109/17477166.2011.604326

35. Hung CS, Lee JK, Yang CY, Hsieh HR, Ma WY, Lin MS, Liu PH, Shih SR, Liou JM, Chuang LM et al: Measurement of visceral fat: should we include retroperitoneal fat? PLOS One 2014, 9(11):e112355.http://dx.doi.org/10.1371/journal.pone.0112355

36. Oelkrug R, Polymeropoulos ET, Jastroch M: Brown adipose tissue: physiological function and evolutionary significance. J Comp Physiol B 2015, 185(6):587606.http://dx.doi.org/10.1007/s00360-015-0907-7

37. Chondronikola M, Volpi E, Børsheim E, Porter C, Annamalai P, Enerbäck S, Lidell ME, Saraf MK, Labbe SM, Hurren NM et al: Brown adipose tissue improves whole-body glucose homeostasis and insulin sensitivity in humans. Diabetes 2014, 63(12):40894099.http://dx.doi.org/10.2337/db14-0746

38. Fisher FM, Kleiner S, Douris N, Fox EC, Mepani RJ, Verdeguer F, Wu J, Kharitonenkov A, Flier JS, Maratos-Flier E et al: FGF21 regulates PGC-1 $\alpha$ and browning of white adipose tissues in adaptive thermogenesis. Genes Dev 2012, 26(3):271281.http://dx.doi.org/10.1101/gad.177857.111

39. Giordano A, Frontini A, Castellucci M, Cinti S: Presence and distribution of cholinergic nerves in rat mediastinal brown adipose tissue. J Histochem Cytochem 2004, 52(7):923930.http://dx.doi.org/10.1369/jhc.3A6246.2004

40. Kotzbeck P, Giordano A, Mondini E, Murano I, Severi I, Venema W, Cecchini MP, Kershaw EE, Barbatelli G, Haemmerle $G$ et al: Brown adipose tissue whitening leads to brown adipocyte death and adipose tissue inflammation. J Lipid Res 2018, 59(5):784- 
794.http://dx.doi.org/10.1194/jlr.M079665

41. Zhang L, Qin Y, Liang D, Li L, Liang Y, Chen L, Tong L, Zhou J, Li H, Zhang H: Association of polymorphisms in LEPR with type 2 diabetes and related metabolic traits in a Chinese population. Lipids Health Dis 2018, 17(1):2.http://dx.doi.org/10.1186/s12944-017-0644$\underline{x}$

42. Behnes M, Brueckmann M, Lang S, Putensen C, Saur J, Borggrefe M, Hoffmann U: Alterations of leptin in the course of inflammation and severe sepsis. BMC Infect Dis 2012, 12:217.http://dx.doi.org/10.1186/1471-2334-12-217

43. Gregor MF, Hotamisligil GS: Inflammatory mechanisms in obesity. Annu Rev Immunol 2011, 29:415-445.http://dx.doi.org/10.1146/annurev-immunol-031210-101322

44. Margetic S, Gazzola C, Pegg GG, Hill RA: Leptin: a review of its peripheral actions and interactions. Int $J$ Obes Relat Metab Disord 2002, 26(11):14071433.http://dx.doi.org/10.1038/sj.ijo.0802142

45. Zhao Z, Peng W, Zhou J, Zhou Y, Liu T, Bai H, Wu Q, Song J, Wu L, Song X et al: Association of LEPR polymorphisms with predisposition and inflammatory response in antituberculosis drug-induced liver injury: A pilot prospective investigation in Western Chinese Han population. Infect Genet Evol 2019, 75:103970.http://dx.doi.org/10.1016/j.meegid.2019.103970

46. Chamberlain G, Fox J, Ashton B, Middleton J: Concise review: mesenchymal stem cells: their phenotype, differentiation capacity, immunological features, and potential for homing. Stem Cel/s 2007, 25(11):2739-2749.http://dx.doi.org/10.1634/stemcells.2007$\underline{0197}$

47. Harvey N, Dennison E, Cooper C: Osteoporosis: impact on health and economics. Nat Rev Rheumato/ 2010, 6(2):99-105.http://dx.doi.org/10.1038/nrrheum.2009.260

48. Lacombe J, Karsenty G, Ferron M: In vivo analysis of the contribution of bone resorption to the control of glucose metabolism in mice. Mol Metab 2013, 2(4):498504.http://dx.doi.org/10.1016/j.molmet.2013.08.004

49. Schwetz $V$, Pieber $T$, Obermayer-Pietsch B: The endocrine role of the skeleton: background and clinical evidence. Eur J Endocrinol 2012, 166(6):959967.http://dx.doi.org/10.1530/eje-12-0030

50. Hess R, Pino AM, Ríos S, Fernández M, Rodríguez JP: High affinity leptin receptors are present in human mesenchymal stem cells (MSCs) derived from control and osteoporotic donors. J Cel/ Biochem 2005, 94(1):50-57.http://dx.doi.org/10.1002/jcb.20330

51. Chang YJ, Shih DT, Tseng CP, Hsieh TB, Lee DC, Hwang SM: Disparate mesenchymelineage tendencies in mesenchymal stem cells from human bone marrow and umbilical cord blood. Stem Cells 2006, 24(3):679-685.http://dx.doi.org/10.1634/stemcells.2004$\underline{0308}$

52. Wang J, Huang R, Xu Q, Zheng G, Qiu G, Ge M, Shu Q, Xu J: Mesenchymal Stem CellDerived Extracellular Vesicles Alleviate Acute Lung Injury Via Transfer of miR-27a-3p. Crit Care Med 2020, 48(7):e599-e610.http://dx.doi.org/10.1097/ccm.0000000000004315

53. Cui Q, Xing J, Yu M, Wang Y, Xu J, Gu Y, Nan X, Ma W, Liu H, Zhao H: Mmu-miR-185 depletion promotes osteogenic differentiation and suppresses bone loss in osteoporosis through the Bgn-mediated BMP/Smad pathway. Cell Death Dis 2019, 10(3):172.http://dx.doi.org/10.1038/s41419-019-1428-1 


\section{Figure legends}

Figure 1. MDP regulates diet-induced glucose homeostasis and adipogenesis.

(A and B) Experimental design for MDP administration of a HFD (A) and LFD (B).

(C and D) Change in blood glucose level of HFD-fed (C) and LFD-fed (D) male mice treated with MDP for 10 days. $n=3-5$ mice for each group.

(E and F) Bodyweight evaluation of HFD-fed (E) and LFD-fed (F) male mice treated with MDP for 10 days. $n=3-5$ mice for each group.

(G) Representative staining (hematoxylin and eosin) images of iBAT, scWAT, and vWAT from the differently treated mice (Scale bar, $20 \mu \mathrm{m}$; from 3 mice per group). Data are presented as mean $\pm \mathrm{SD}$.

Figure 2. Lepr participates in the changes of energy metabolism induced by MDP. (A) Heatmap showing differential expression of energy metabolism-related genes in osteocytes after MDP intervention.

(B) and (C) Differential expression of mRNAs in osteocytes based on KEGG and GO databases.

(D) Lepr expression in serum of mice in the four groups.

(E) Representative staining (immunohistochemical) images of iBAT, scWAT, and vWAT from the four groups. Scale bar, $20 \mu \mathrm{m}$.

(F) Staining (immunohistochemical) for Lepr in the femur and alveolar bone in mice of the four groups. Scale bar, $20 \mu \mathrm{m}$. ${ }^{*} \mathrm{P}<0.05$. Data are presented as mean $\pm \mathrm{SD}$ from 3 mice per group.

Figure 3. Effect of MDP on the mass and quality of mice in male mice fed a HFD or LFD.

(A and B) Representative sagittal three-dimensional reconstructions of micro-CT images from the mandible (A) and femur (B) of L-NaCl, L-MDP, H-NaCl, and H-MDP groups.

(C) Bone histomorphometry and images of mice from the four groups by micro-CT. $\mathrm{BV} / \mathrm{TV}$, bone volume/total tissue volume; $\mathrm{BS} / \mathrm{BV}$, bone surface/bone volume; Tb.Sp, trabecular spacing; Tb.Th, trabecular thickness; Tb.N, trabeculae number.

(D) Representative staining (immunohistochemical) images of the femur of mice from the four groups. Scale bar (left), $1000 \mu \mathrm{m}$. Scale bar (right), $500 \mu \mathrm{m} . * \mathrm{P}<0.05$. Data are presented as mean $\pm \mathrm{SD}$ from 3 mice per group.

Figure 4. miR-384-5p contained Lepr targets and modulated Lepr expression in vitro.

(A) Prediction using bioinformatics analysis of the target site of mmu-miR-384-5p with Lepr-3'UTR applied by TargetScan.

(B) Sequencing results for Lerp-3'UTR (WT) and mut-Lepr-3'UTR (MUT). The red rectangle represents the binding site in WT and core mutation site in MUT.

(C) The luciferase expression vector psiCHECK-2 was used to detect the activity of the luciferase reporter gene.

(D) Dual luciferase reporter assay in HEK293T cells. ${ }^{*} \mathrm{P}<0.05$.

(E) During the osteogenic differentiation of BMSCs, Lepr expression was upregulated, but miR-384-5p expression was downregulated. ${ }^{*} \mathrm{P}<0.05$.

(F) Expression of miR-384-5p and Lepr mRNA in BMSCs after transfection. ${ }^{*} \mathrm{P}<0.05$. 
Figure 5. miR-384-5p affected osteogenic differentiation of BMSCs through regulation of Lepr expression.

(A) Representative images of alkaline phosphatase (ALP) and Oil Red O staining of BMSCs induced by the supernatants of osteocytes with HG or LG medium after MDP intervention.

(B) Protein expression of Lepr induced by the supernatants of osteocytes with HG or LG medium after MDP intervention. ${ }^{*} \mathrm{P}<0.05$. (C) Changes in expression of Lepr and miR-384-5p mRNA induced by the supernatants of osteocytes with HG or LG medium after MDP intervention. $* \mathrm{P}<0.05$. 
A

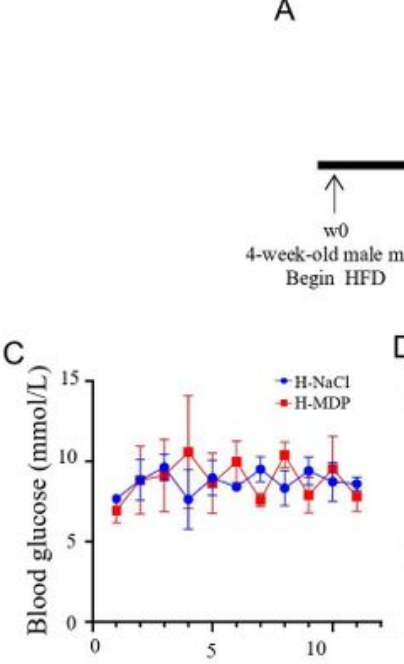

Saline or 2 ug MDP

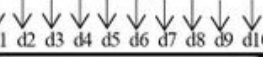

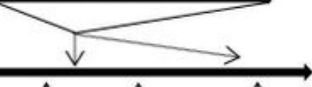

HFD Model

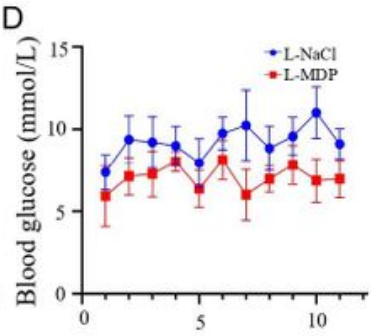

B

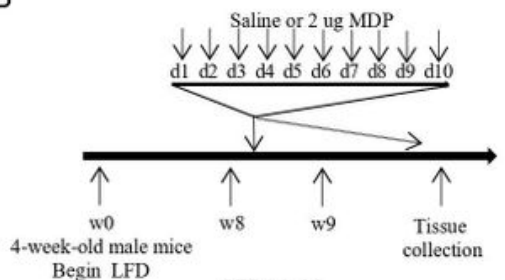

LFD Model
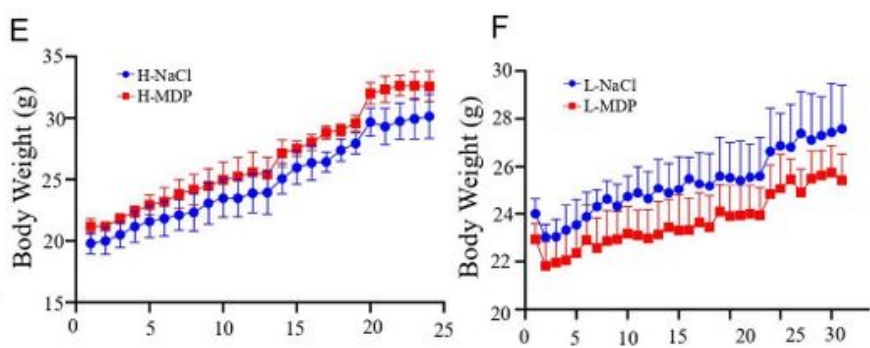

G

iBAT

scWAT

vWAT

$\mathrm{L}-\mathrm{NaCl}$
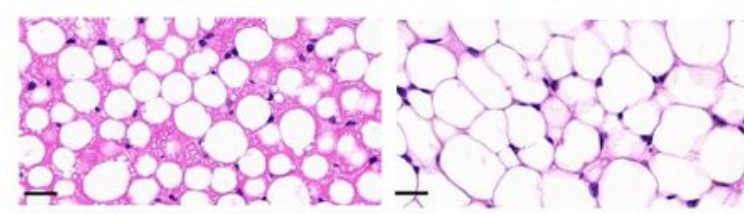

L-MDP
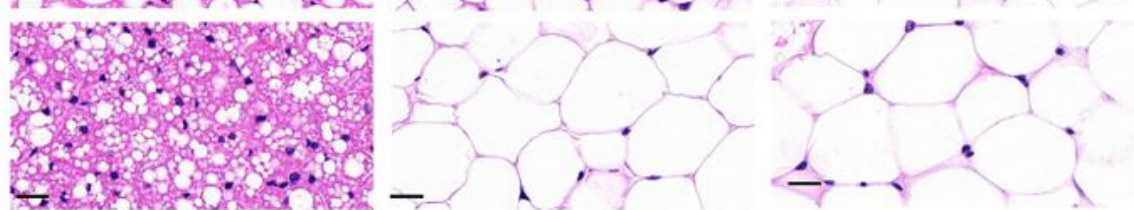

$\mathrm{H}-\mathrm{NaCl}$
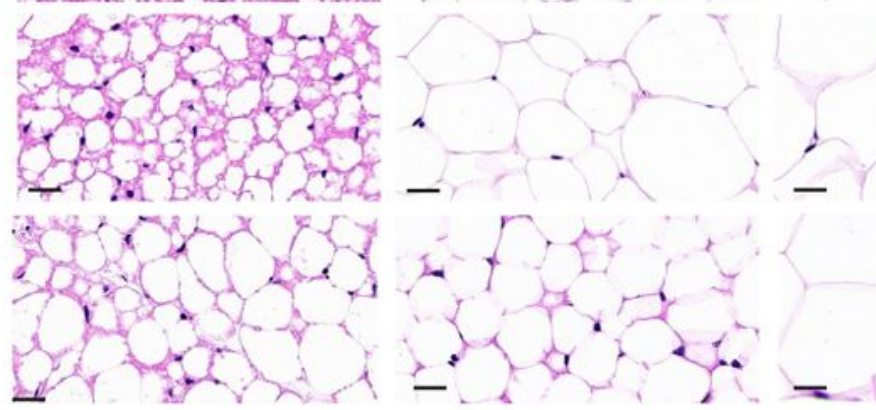

Fig.1 MDP regulates diet-induced glucose homeostasis and adipogenesis

(A and B) Experimental design for MDP administration of HFD (A) and LFD feeding (B).

(C and D) Blood glucose change of HFD-fed(C) and LFD-fed(D) male mice treated with MDP for 10 days. $\mathrm{n}=3-5$ mice for each group.

( $\mathrm{E}$ and F) Body weight evaluation of HFD-fed(E) and LFD-fed(F) male mice treated with MDP for 10 days. $\mathrm{n}=3-5$ mice for each group.

G) The representative hematoxylin and eosin staining images of iBAT, scWAT, and vWAT from the differently treated mice (Scale bar,20 $\mu \mathrm{m}$; from three mice per group). Data are presented as mean $\pm \mathrm{SD}$.

\section{Figure 1}

Please See image above for figure legend. 

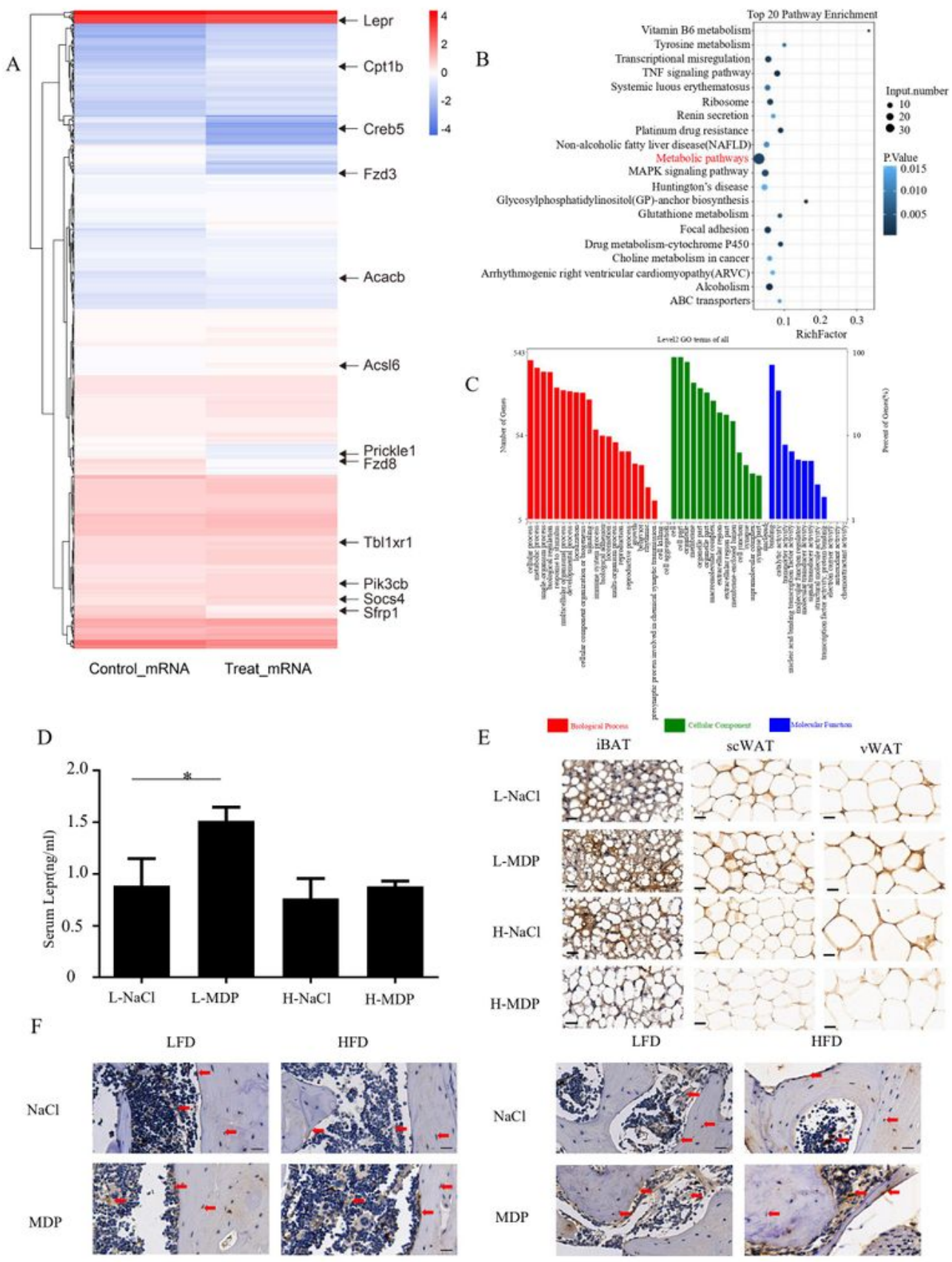

Fig. 2 Lepr participates in the changes of energy metabolism induced by MDP

(A)The heatmap of differential expression of energy metabolism related genes in osteocytes after MDP intervention.

(B) and (C) KEGG and GO analyses of differentially expressed mRNAs in osteocytes .

((D) Serum Lepr level of the four groups

(E) The representative Immunohistochemical staining images of iBAT, scWAT, and vWAT from four groups. Scale bar,20um

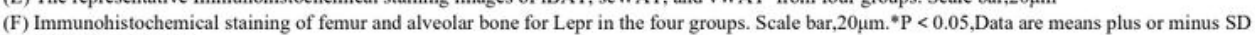
from 3 mice per group.

\section{Figure 2}

Please See image above for figure legend. 

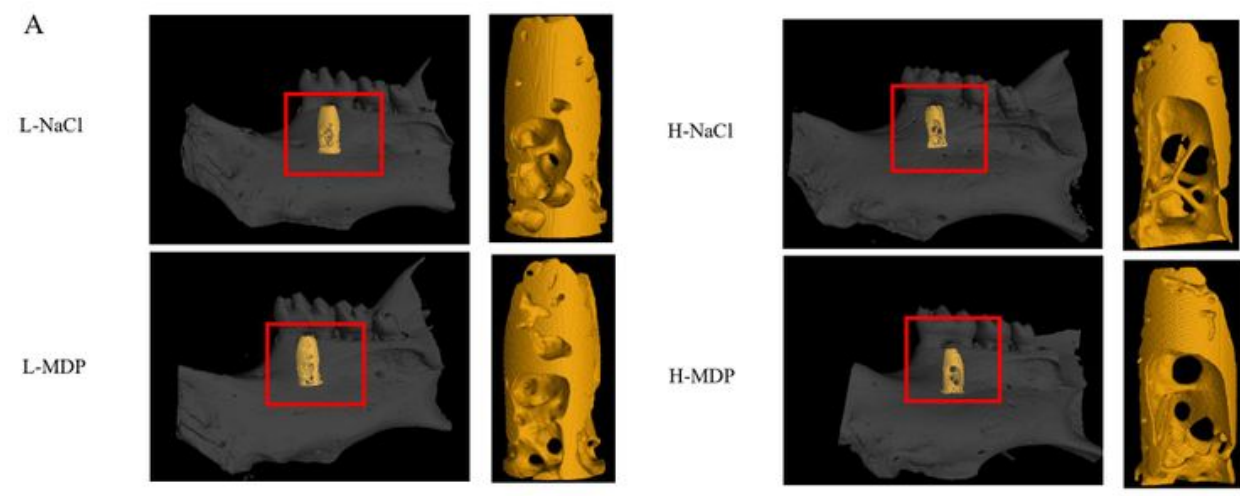

B
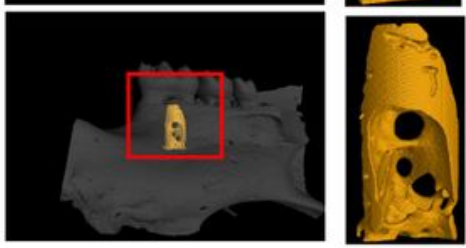

L-MDP

L-NaCL

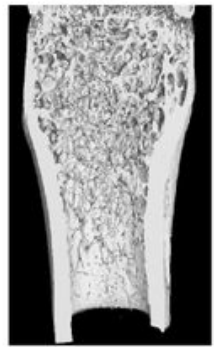

$\mathrm{C}$
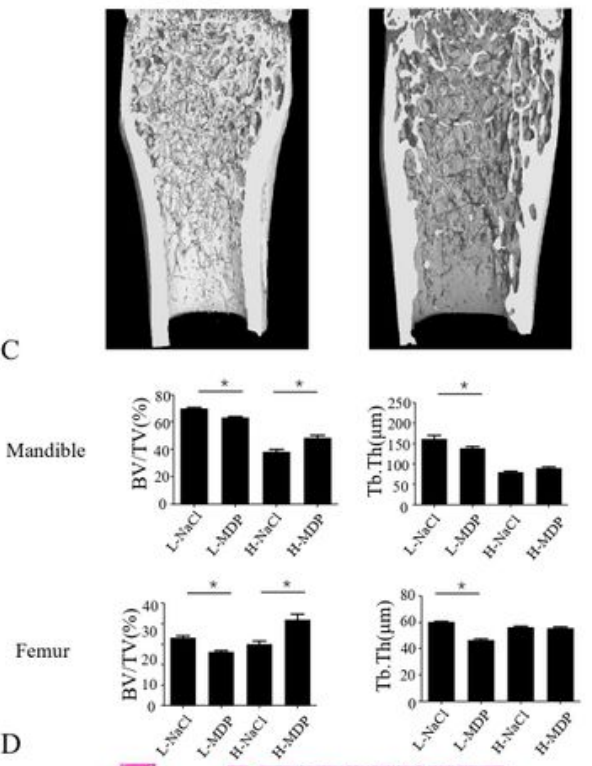

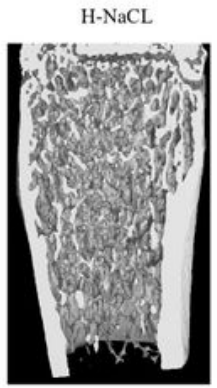

H-MDP
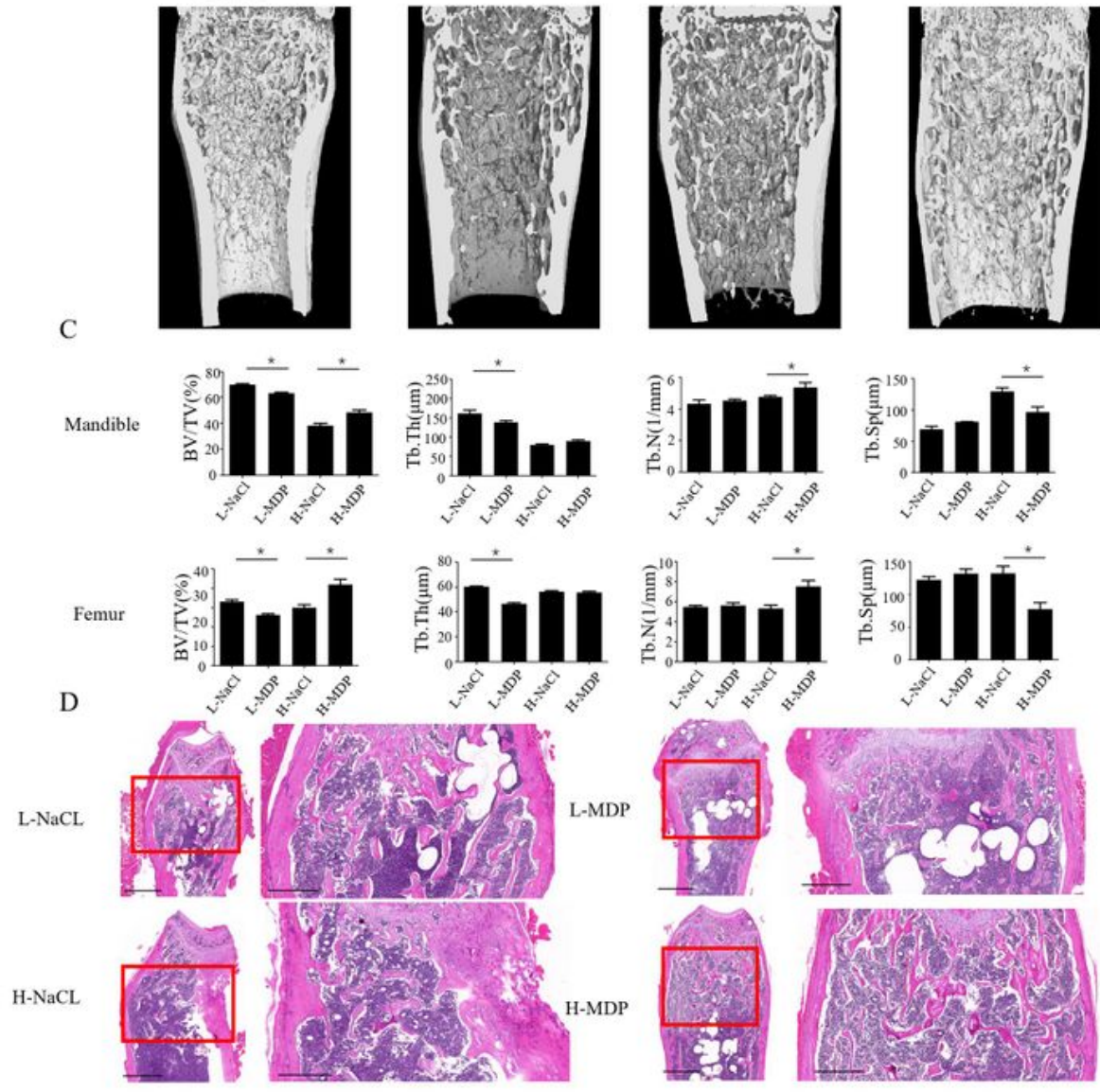

Fig.3. Effect of MDP on bone mass and quality in male mice placed on HFD or LFD

$\mathrm{A}$ and $\mathrm{B}$. Representative sagittal three-dimensional reconstructions of micro-CT images from mandible(A) and femur(B) of L-NaCl, L-MDP,H-NaCl and H-MDP groups

C. Bone histomorphometric analyses and images of the four groups by $\mu \mathrm{CT}$. Abbreviations: BV/TV,bone volume/total tissue volume; BS/BV,bone surface/bone volume; Tb.Sp, trabecu lar spacing; Tb.Th, trabecu lar thickness; $\mathrm{Tb} . \mathrm{N}$, trabecular number.

D. The representative Immunohistochemical staining images of femur from four groups. Scale bar(left), $1000 \mu \mathrm{m}$, Scale bar(right),500 $\mu \mathrm{m} . .^{*} \mathrm{P}<0.05$,Data are means plus or minus SD from 3 mice per group.

\section{Figure 3}

Please See image above for figure legend. 
A

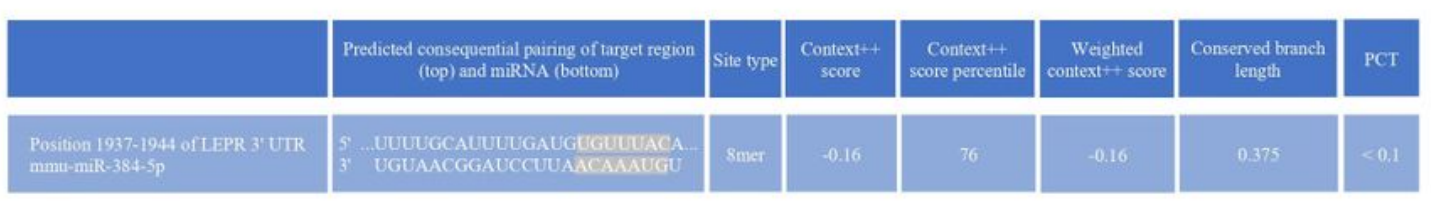

B

Lepr-3 UTR Mut-Lepr-3 UTR

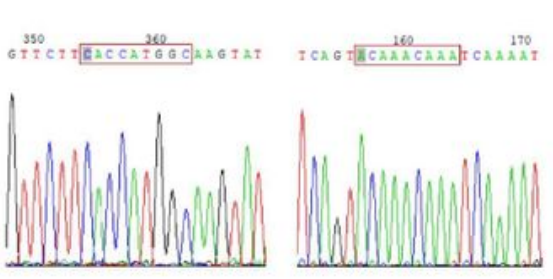

C

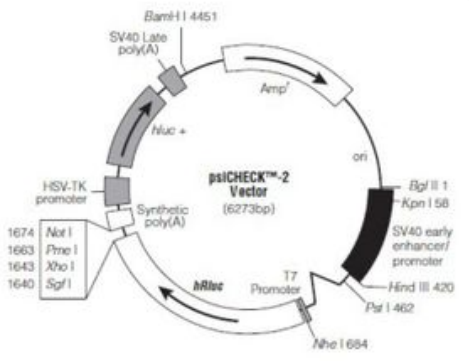

E

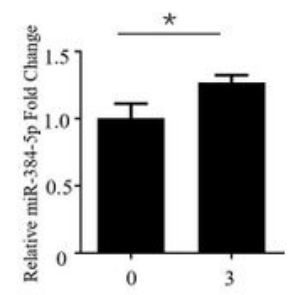

D

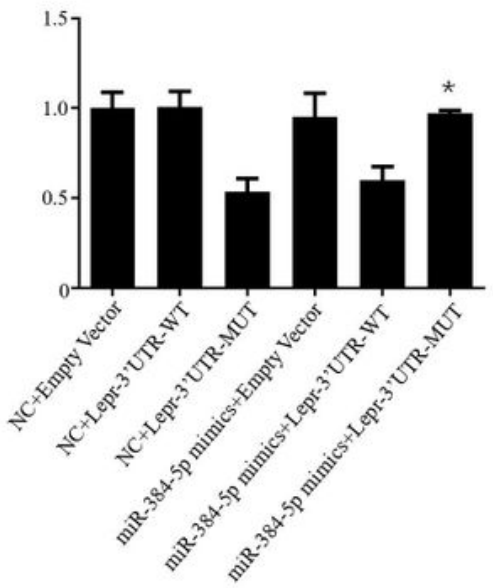

F
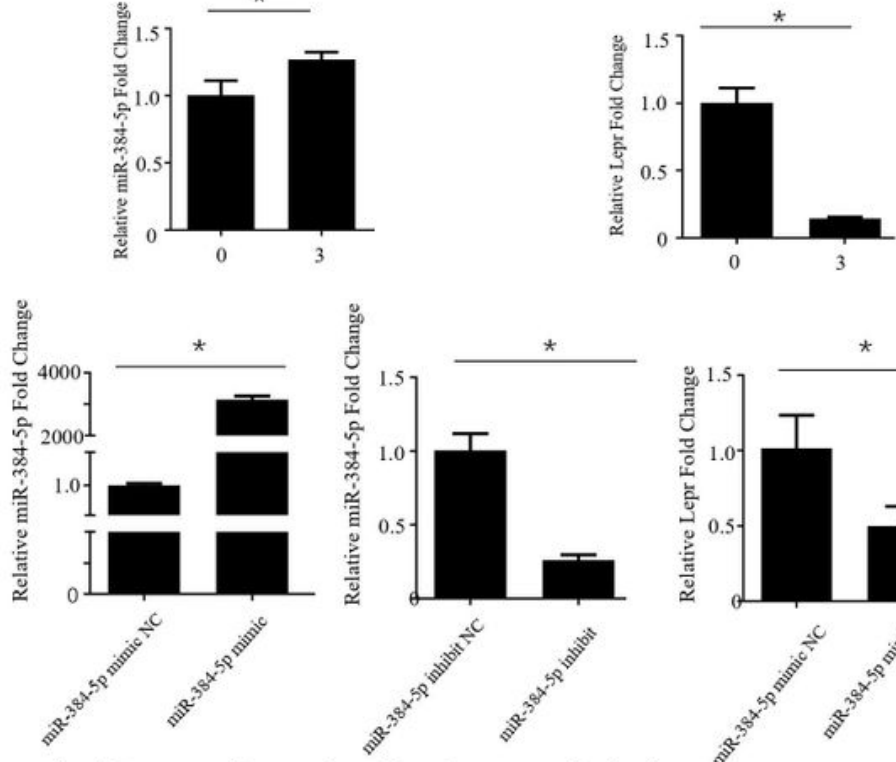

Fig.4 miR-384-5p contained the targets of Lepr and modulates Lepr expression in vitro

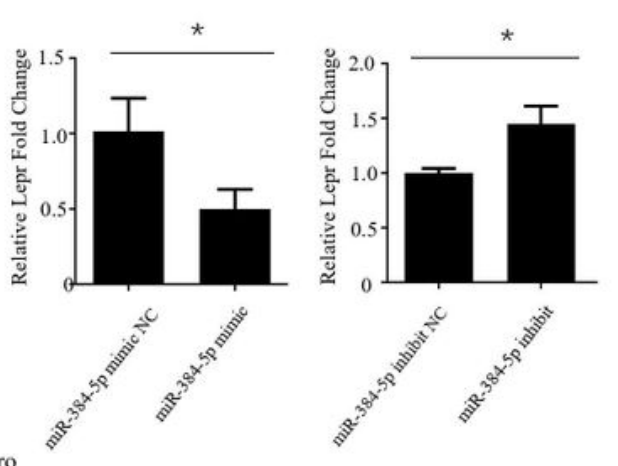

A. Bioinformatic prediction of the target site of mmu-miR-384-5p with Lepr-3'UTR applied by Target Scan.

B. Sequencing results for Lerp-3'UTR (WT) and mut-Lepr-3'UTR (MUT). The red rectangle represents the binding site in WT and the core mutation site in MUT.

C. Luciferase expression vector, psiCHECK-2,was used to detect the activity of luciferase reporter gene.

D. Dual luciferase reporter assay in HEK293T cells. ${ }^{*} \mathrm{P}<0.05$.

E. During the osteogenic differentiation of BMSC, the expression of Lepr was up-regulated, but miR-384-5p expression was down-regulated . $* \mathrm{P}<0.05$.

F. Expression of miR-384-5p and Lepr mRNA in BMSC cells after transfection. ${ }^{*} \mathrm{P}<0.05$.

Figure 4

Please See image above for figure legend. 
A

L-NC

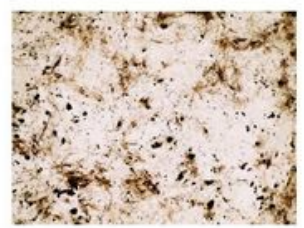

L-MDP

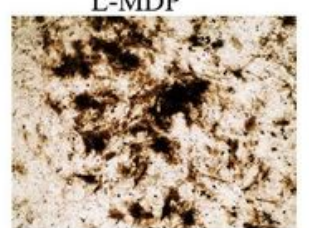

$\mathrm{H}-\mathrm{NC}$

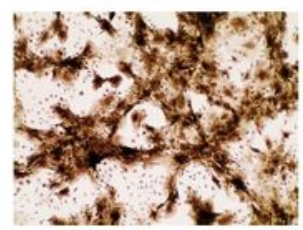

H-MDP

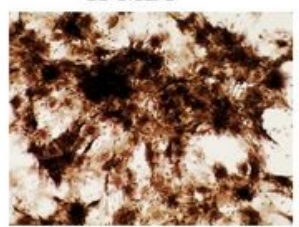

L-NC

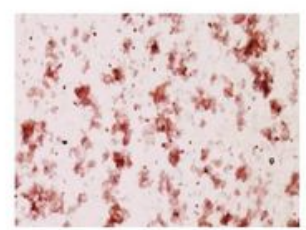

L-MDP

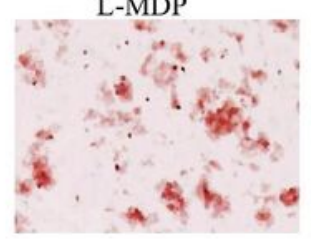

$\mathrm{H}-\mathrm{NC}$

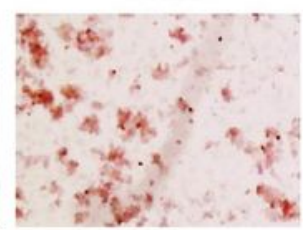

H-MDP

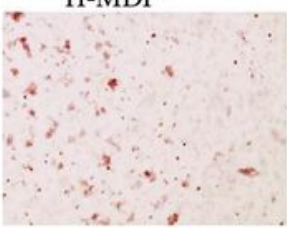

B

L-MDP L-NC H-MDP H-NC

Lepr

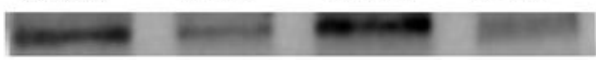

$\beta$-ACTIN
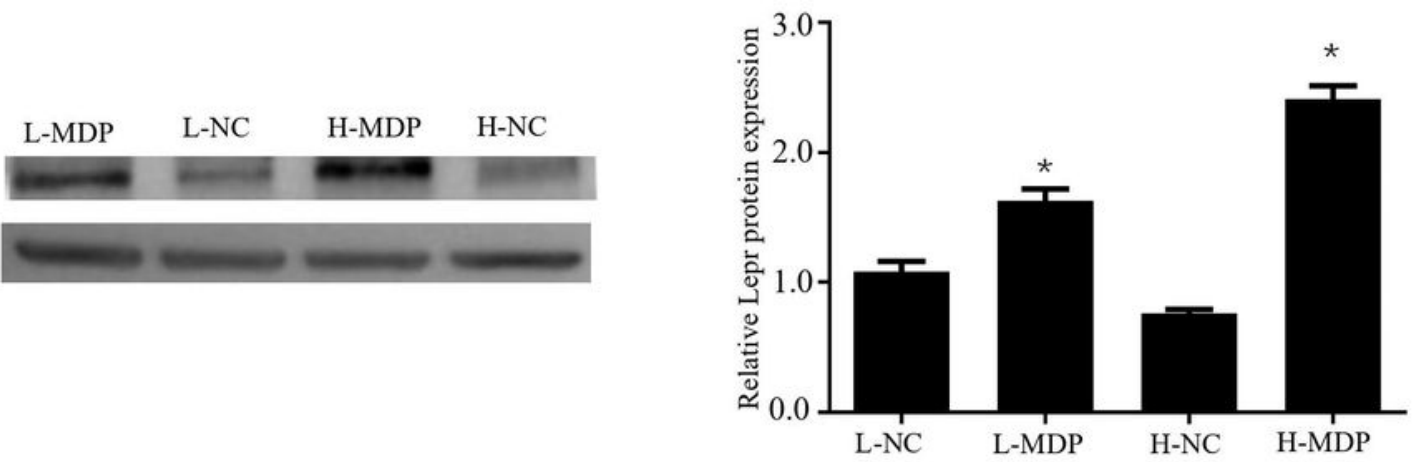

C
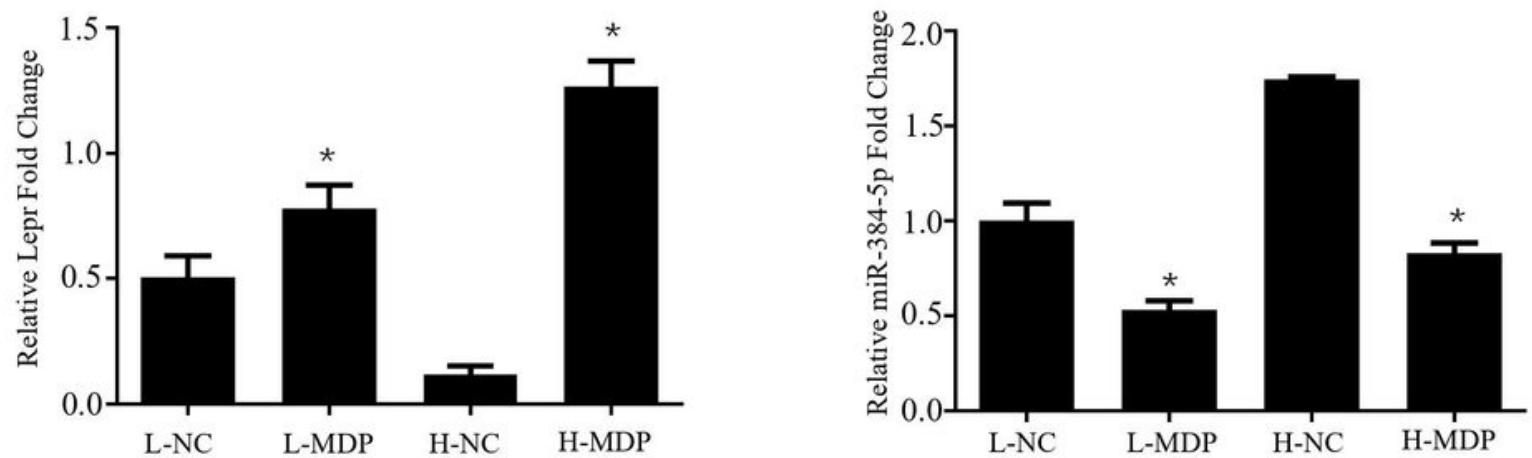

Fig.5 miR-384-5p affected osteogenic differentiation of BMSC through regulating Lepr

A. Representative images of alkaline phosphatase(ALP) and oil red o staining of BMSC cells induced by the supernatant of osteocytes with HG or LG medium after MDP intervention

B. Protein level of Lepr induced by the supernatant of osteocytes with HG or LG medium after MDP intervention* $\mathrm{P}<0.05$.

C. Changes in Lepr and miR-384-5p mRNA induced by the supernatant of osteocytes with HG or LG medium after MDP intervention. *P<0.05.

\section{Figure 5}

Please See image above for figure legend. 